\title{
Complement C3a receptor-mediated vascular dysfunction: a complex interplay between aging and neurodegeneration
}

\author{
Kanchan Bhatia, ${ }^{1,2}$ Saif Ahmad, ${ }^{1}$ Adam Kindelin, ${ }^{1}$ and Andrew F. Ducruet ${ }^{1}$ \\ 'Department of Neurosurgery, Barrow Neurological Institute, St. Joseph's Hospital and Medical Center (SJHMC), Dignity Health, Phoenix, Arizona, USA. ${ }^{2}$ School of Mathematical and Natural Sciences, Arizona \\ State University, Phoenix, Arizona, USA.
}

\begin{abstract}
Vascular dysfunction resulting in compromised blood-brain barrier (BBB) integrity is evident in aging and disease. Although the complement $\mathrm{C} 3 \mathrm{a} /$ C3a receptor ( $\mathrm{C} 3 \mathrm{a} / \mathrm{C} 3 \mathrm{aR}$ ) axis influences normal brain aging and disease progression, the mechanisms governing endothelial C3aR-mediated neurovascular inflammation and BBB permeability remain unexplored. In this issue of the $J C I$, Propson et al. investigated endothelial $\mathrm{CBa} \mathrm{J}$ C3aR signaling in normal, aged, and neurodegenerative mouse models. Endothelial C3aR signaling modulated age-dependent increases in VCAM1, initiated peripheral lymphocyte infiltration, and enhanced microglial activity. Increased calcium release downstream of C3aR signaling disrupted the vascular endothelial cadherin (VE-cadherin) junctions, increased BBB permeability, and degraded vascular structure and function. Mice lacking C3aR ( $\left.\mathrm{C3ar1}^{-/-}\right)$and mice treated with a C3aR antagonist showed attenuated age-related microglial reactivity and neurodegeneration. These results confirm that complement-mediated signaling impacts vascular health and BBB function in normal aging and neurodegenerative disease, suggesting that complement inhibitors represent a therapeutic option for cerebral microvascular dysfunction.
\end{abstract}

\section{The blood-brain barrier and cerebral microvascular dysfunction}

The estimated human life expectancy has increased worldwide, and by the year 2050 the population of persons aged 60 years and above will double. Moreover, the population for those 80 years and above will triple (1). This dramatic projected increase in the elderly population will be accompanied by a rise in the number of patients affected by age-related neurological diseases, contributing to tremendous socioeconomic costs. Thus, there is a pressing need to develop approaches to prevent and treat disease, and improve quality of life in the aged population. The natural aging process induces neurodegenerative disorders due to a decline in the structure and function of the brain. Age-dependent cognitive impairment that is observed in otherwise healthy individuals is often associated with structural and functional changes of the blood-brain barrier (BBB) and exacerbated by subtle pathological changes observed in the onset of Alzheimer's disease. Thus, normal aging may enhance the molecular mechanisms of neurodegenerative disease.

The limited permeability of the BBB to macromolecules is established during development and maintained in adult-
Related Article: https://doi.org/10.1172/JCI140966

Conflict of interest: The authors have declared that no conflict of interest exists.

Copyright: @ 2021, American Society for Clinical Investigation.

Reference information: J Clin Invest. 2021;131(1):e144348. https://doi.org/10.1172/JCI144348.

hood by surrounding pericytes, smooth muscle cells, astrocytes, microglia, and neurons (2-4). Brain endothelial cells (BECs) also play an important role in regulation of barrier function, transport function, and immune surveillance in the brain. BECs have a specialized phenotype that is greatly influenced by their local environment and interaction with components of the neurovasculature. Tight junction protein complexes expressed by BECs prevent the diffusion of substances between cells and limit lateral diffusion of membrane proteins, thus conferring BEC polarity. Age-dependent alterations in the tight junction neurovascular components eventually compromise barrier function. Moreover, since endothelial dysfunction promotes morbidity and mortality in age-related disease, maintaining the correct function of this vascular layer is an essential determinant of healthy aging (5).

In this issue of the JCI, Propson et al. (6) report increased VCAM1 expression in endothelial cells of the cortex and hippocampus in aged mice. The authors revealed age-associated changes in BBB integrity. Notably, in both large vessels and capillaries, calcium $\left(\mathrm{Ca}^{2+}\right)$ release from the endothelial cells caused downregulation of vascular endothelial cadherin (VE-cadherin) intercellular junctions (Figure 1). Furthermore, Propson and researchers found reduced expression of Ocln, Tjp1, and Cldn 5 mRNAs, reflecting impaired junctional components of the BBB. Importantly, elevated surface expression of vascular cell adhesion molecule 1 (VCAM1) on BECs, occurring both with normal aging and in neuronal diseases, led to microglial cell activation and cognitive deficits via a neuroinflammatory process (6).

Age- and disease-related changes in the immune system Changes in the immune system have long been recognized to occur with normal 


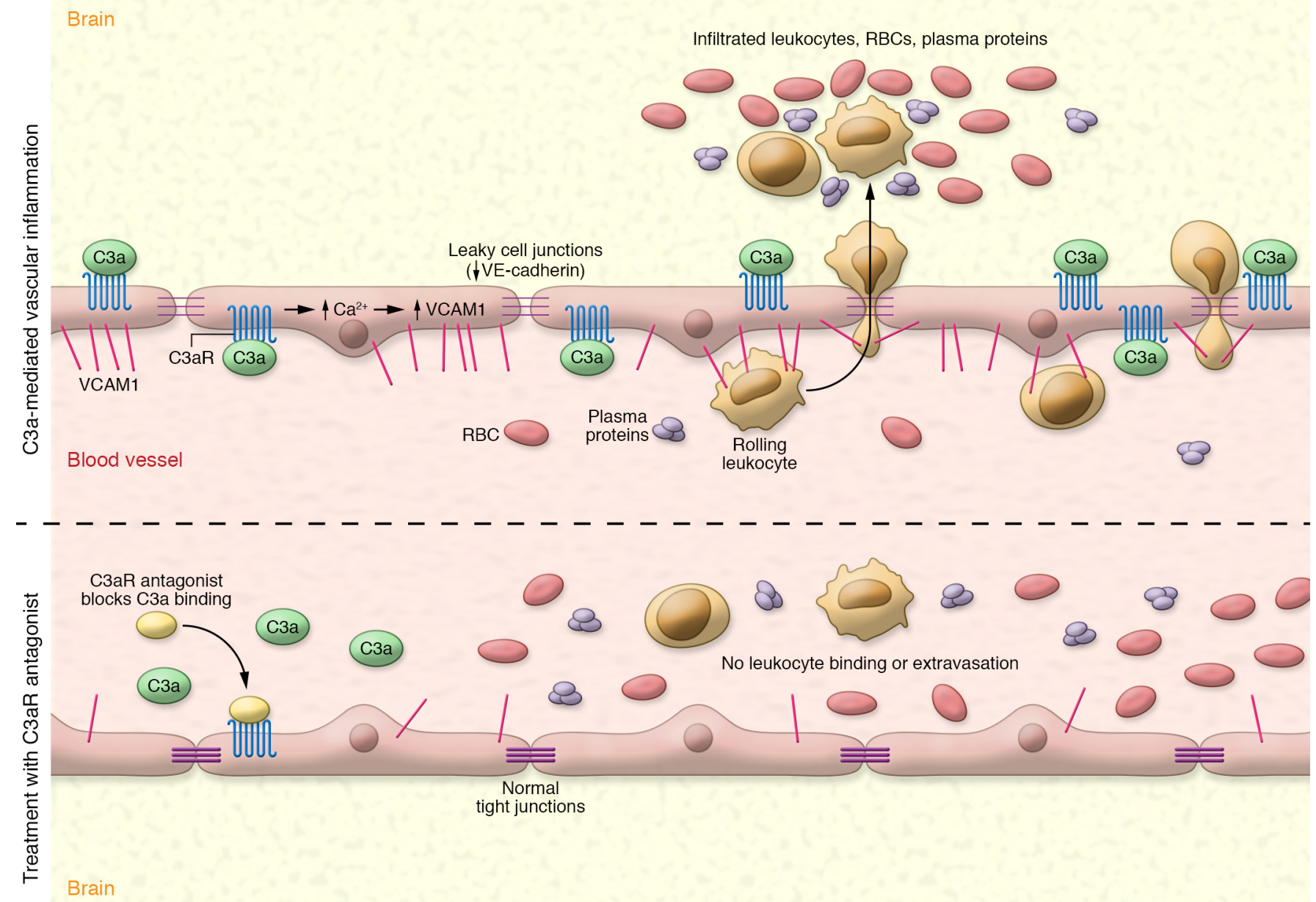

Figure 1. Model for C3a-mediated neurovascular inflammation and BBB permeability. Top: With age, C3aR-mediated endothelial cell VCAM1 expression increases in the cortex and hippocampus and VE-cadherin is downregulated. Subsequent endothelial cell calcium (Ca ${ }^{2+}$ ) release disrupts tight junctions, which increases BBB permeability. RBCs, plasma proteins, and leukocytes can infiltrate the vasculature where they can adhere, roll, and then leak into extravascular tissue. Bottom: Propson et al. (6) showed that treatment with a C3aR antagonist blocked C3a from binding to C3aRs, preventing pathological changes in tight junctions and subsequent leukocyte extravasation.

aging and neuroinflammation plays a central role in diverse neurological diseases (7). However, it is less well understood how specific age-related changes in the immune system affect CNS function in the disease setting. The adaptive and innate immune systems mount initial protective responses against infections and facilitate the rapid recognition and neutralization of specific pathogens upon subsequent exposure, playing a role in both sterile tissue injury and wound repair. The complement pathway is a critical regulator of innate immunity (8) that has been implicated in aging and disease. Complement activation requires the cleavage of the central complement factor C3 to C3a and C3b, which elicit downstream events through binding to their receptors $\mathrm{C} 3 \mathrm{aR}$ and $\mathrm{CR} 3$, respective- ly (9). Signaling via C3a/C3aR has been implicated in various disease conditions, including Alzheimer's disease where C3a/C3aR mediates neuron-immune crosstalk that influences network function and $A \beta$ pathology (10). C3a is an anaphylatoxin that drives proinflammatory signaling and chemotaxis via binding to $\mathrm{C} 3 \mathrm{aR}$, a receptor present in neurons, microglia, astrocytes, and vascular BECs $(3,11)$. Propson et al. demonstrate that C3a cleaved from astrocytic C3 interacted with endothelial $\mathrm{C} 3 \mathrm{aR}$ at the $\mathrm{BBB}$, promoting an increase in VCAM1 expression. VCAM1 is a surface protein in the adhesion molecule family that regulates immune cell processes such as rolling, adhesion, and extravasation. Propson and investigators found that endothelial C3aassociated VCAM1 expression increased
$\mathrm{BBB}$ permeability in aged mouse brains as well as in brains of a tau-transgenic mouse model (PS19), leading to an increase in peripheral $\mathrm{CD}^{+} \mathrm{T}$ cell infiltration into the brain parenchyma (6). The expansion of $\mathrm{CD} 8^{+} \mathrm{T}$ cells within the brain can promote microglial activation, a pathway that has also been observed in brain tissue of patients with both Parkinson's and Alzheimer's disease (12). Aberrant microglial activation further aggravates tissue injury by promoting white matter injury and cognitive deficits due to altered cerebral blood flow (13). These observations add to the growing number of studies suggesting that many neurological diseases and conditions could be the result of normal, age-related vascular dysfunction, which Propson et al. show is exacerbated by C3a/C3aR dysregulation of the BBB (6). 


\section{Attenuating age-related changes in the cerebral microvasculature}

Global targeting of C3aR by C3ar1 deletion and antagonist inhibition attenuated age-related changes in VCAM1 and the cerebral microvasculature. However, to tease out the role specifically of endothelial C3aR in mediating the effects on the BBB as well as morphological changes in the brain with aging, Propson et al. generated mice with a conditional endothelial cell-specific C3ar1 deletion by crossing C3arl-floxed mice with Tie2-Cre mice. These conditional knockouts demonstrated suppressed VCAM1 expression, attenuated changes in vessel morphology, and reduction in aberrant activation and influx of peripheral immune cells with aging to the same degree as with global ablation/ pharmacological targeting of $\mathrm{C} 3 \mathrm{aR}$, providing further evidence of a role for endothelial cell-specific $\mathrm{C} 3 \mathrm{aR}$ in regulating brain health in aging. Furthermore, morphological changes in capillary cross-section area and overall incidence of tortuous vessel segments in the hippocampus can contribute to neurodegenerative disease, which can be mitigated by genetic ablation of C3ar1. For example, in age-related models of Alzheimer's disease, C3aR deletion attenuates neuroinflammation and reduces synaptic deficits and neurodegeneration in the PS19 tau model (10). Thus, pharmacological targeting of the $\mathrm{C} 3 \mathrm{aR}$ in age-related neurodegenerative diseases is the next logical step. Propson et al. (6) highlight the potential of strategies targeting the complement $\mathrm{C} 3 \mathrm{a} / \mathrm{C} 3 \mathrm{aR}$ axis as a means of preventing and treating age-related neurodegenerative disease.

\section{Acknowledgments}

AFD receives research funding from the Barrow Neurological Foundation.

Address correspondence to: Andrew F. Ducruet, Department of Neurosurgery, Barrow Neurological Institute, St. Joseph's Hospital and Medical Center (SJHMC), Dignity Health, 350 W Thomas Rd, Phoenix, Arizona 85013, USA. Phone: 602.406.3964; Email: andrew.ducruet@ barrowbrainandspine.com.

1. Bejot Y, Yaffe K. Aging population: a neurological challenge. Neuroepidemiology. 2019;52(1-2):76-77.

2. Chen MB, et al. Brain endothelial cells are exquisite sensors of age-related circulatory cues. Cell Rep. 2020;30(13):4418-4432.

3. Ahmad S, et al. The role of complement C3a receptor in stroke. Neuromolecular Med.
2019;21(4):467-473.

4. Haddad-Tovolli R, et al. Development and function of the blood-brain barrier in the context of metabolic control. Front Neurosci. 2017;11:224.

5. El Assar M, et al. Mechanisms involved in the aging-induced vascular dysfunction. Front Physiol. 2012;3:132.

6. Propson NE, et al. Endothelial C3a receptor mediates vascular inflammation and BBB permeability during aging. J Clin Invest. https://doi. org/10.1172/JCI140966.

7. Ransohoff RM. How neuroinflammation contributes to neurodegeneration. Science. 2016;353(6301):777-783.

8. Veerhuis R, et al. Complement in the brain. $\mathrm{Mol}$ Immunol. 2011;48(14):1592-1603.

9. Dunkelberger JR, Song WC. Complement and its role in innate and adaptive immune responses. Cell Res. 2010;20(1):34-50.

10. Litvinchuk A, et al. Complement C3aR inactivation attenuates tau pathology and reverses an immune network deregulated in tauopathy models and Alzheimer's disease. Neuron. 2018;100(6):1337-1353.

11. Luchena $\mathrm{C}$, et al. Contribution of neurons and glial cells to complement-mediated synapse removal during development, aging and in Alzheimer's disease. Mediators Inflamm. 2018;2018:2530414.

12. Schetters STT, et al. Neuroinflammation: microglia and $\mathrm{T}$ cells get ready to tango. Front Immunol. 2017;8:1905.

13. Zhang LY, et al. Microglia exacerbate white matter injury via complement C3/C3aR pathway after hypoperfusion. Theranostics. 2020;10(1):74-90. 